\title{
Examen Nacional de Odontología en Chile
}

\section{National exam of dentistry of Chile}

1. Universidad La Frontera. Temuco, Chile.

2. Universidad de Chile. Santiago, Chile.

*Correspondencia a: Ramón Fuentes | Dirección: Av. Francisco Salazar 01145, Temuco, Chile. | E-mail: ramon.fuentes@ufrontera.cl Trabajo recibido 04/10/2021

Aprobado para su publicación 25/10/2021

\author{
Ramón Fuentes $^{1 *}$, Jorge Gamonal ${ }^{2}$
}

El 21 de octubre del año 2019 se realizó, en las dependencias del Colegio de Dentistas de Chile, el acto de lanzamiento del Examen Nacional Odontológico (ENO) que organizó la Red Estatal de Odontología de Chile con el fuerte y decidido apoyo de la Directiva del colegio de la orden. Recordamos, que el lunes 26 de noviembre del año 2018 se llevó a cabo el piloto del ENO, de tal forma que desde las 08.30 horas y en forma simultánea los/as estudiantes de las Facultades y Escuelas de Odontología de las Universidades Estatales, que están próximos a egresar rindieron este examen. La Red Estatal de Odontología (REO), conformada por la Universidad de Chile, Universidad de Valparaíso, Universidad de la Frontera, Universidad de Talca, Universidad de Antofagasta, Universidad de la Serena y Universidad Arturo Prat, lograba en coordinación con el Colegio de Dentistas un extraordinario logro en el ámbito de la formación del pregrado, la existencia de un examen final, único para todas las universidades estatales en primera instancia.

Con esta iniciativa se buscaba complementar la acreditación del pregrado, que evalúa los procesos que cada universidad tiene implementado en la formación de pregrado principalmente, con una evaluación individual de cada egresado, para determinar si este cumplía con los mínimos exigibles en relación a sus competencias de egreso para su correcto desempeño profesional(1).

En preparación de este examen las universidades integrantes de la REO realizaron diversas actividades, como conocer el inicio, historia y presente del EUNACOM (Examen Único Nacional de Conocimientos de Medicina), para lo cual se contó con la presencia del Dr. Beltrán Mena, que participa desde los inicios de ese examen. Se realizó una coordinación a través de las carreras y/o escuelas de Odontología de las Universidades estatales para desarrollar las preguntas que formarían parte de este examen. Se concordaron las áreas de formación de la Odontología que se evaluarían en el primer examen, que era un piloto. Se redactaron las preguntas con orientaciones de forma y fondo y cada escuela o carrera debía integrar en la creación de estas preguntas a las asignaturas, disciplinas o cátedras a su cargo, para poder consensuar adecuadamente el instrumento.

Finalmente, luego de un arduo trabajo de parte de los docentes que participaron en el desarrollo del examen, se logró tener uno en condiciones para ser aplicado a fines del año 2019. La aplicación de este examen, su logística demostró que cuando hay interés en una causa superior, es posible lograr los objetivos. Se contó con la ayuda de las siete universidades de la Red Estatal y del Colegio de Dentistas para su ejecución.

La intención era continuar con este instrumento, perfeccionándolo en forma y fondo, sociabilizándolo más en las comunidades educativas odontológicas de cada universidad y entre el gremio en general. Lamentablemente esto no continuó.

Si bien es cierto la pandemia afectó en nuestras diversas actividades académicas, a nuestro juicio, el Examen Nacional de Odontología no debió suspenderse. Tengamos presente que el EUNACOM se siguió desarrollando pese a las dificultades, y que somos una carrera de acreditación obligatoria. Y que relevancia hubiese ido tomando el Examen Nacional de Odontología, durante estos años de pandemia, para asegurar y dar fe pública que no solo nuestros procesos de formación de pregrado son de calidad (acreditación de carreras) sino que velamos por el cumplimiento individual de competencias teóricas de los egresados aún en tiempos de pandemia.

Hacemos un llamado para retomar esta iniciativa y así aportar con instrumentos claro y objetivos en la calidad de la formación de nuestros Cirujanos-Dentistas.

\section{Bibliografía}

1.-Fuentes J, Silva M, Llermaly S. Examen nacional de odontología. Revisión biblio-

gráfica de instrumentos. Int J Odontostomat. 2014; 8(1):125-31. 